\title{
Influência de um Protocolo de Mobilização Precoce no comportamento autonômico de pacientes submetidos a Angioplastia Coronária Transluminal Percutânea
}

\author{
Influence of an Early Mobilization Protocol on the Autonomic Behavior of Patients Undergoing Percutaneous \\ Transluminal Coronary Angioplasty
}

\author{
Bárbara Oliveira Silveira, ${ }^{1 \oplus}$ Jade Lara de Melo, ${ }^{1 \oplus}$ Graziella Paula de Oliveira Neri, ${ }^{1 \oplus}$ Michele Lima Gregório, ${ }^{2}$ \\ Moacir Fernandes de Godoy, ${ }^{3 \oplus}$ Marilita Falangola Accioly ${ }^{1}$ \\ Universidade Federal do Triângulo Mineiro, ${ }^{1}$ Uberaba, MG - Brasil \\ Faculdade de Medicina de São José do Rio Preto (FAMERP) - Pós Graduação Enfermagem, ${ }^{2}$ São Jose do Rio Preto, SP - Brasil \\ Faculdade de Medicina de São José do Rio Preto (FAMERP) - Cardiologia e Cirurgia Cardiovascular, ${ }^{3}$ São Jose do Rio Preto, SP - Brasil
}

\section{Resumo}

Fundamento: Gráficos de recorrência (GR) permitem uma análise não linear da variabilidade de frequência cardíaca (VFC) e fornecem informações sobre o sistema nervoso autônomo (SNA).

Objetivos: Avaliar se a mobilização precoce em pacientes submetidos a angioplastia coronária transluminal percutânea (ACTP) influencia os componentes quantitativos e qualitativos dos GR.

Métodos: Um total de 32 participantes que foram submetidos a ACTP foram divididos entre um grupo de controle (GC - sem exercícios físicos) e grupo de mobilização precoce (GMP - com exercícios físicos) A frequência cardíaca batimento a batimento foi registrada utilizando um cardiofrequencímetro em ambos os grupos na admissão e na alta. Os índices lineares nos domínios de tempo e frequência foram analisados, bem como os índices não lineares obtidos pelos GR. $O$ protocolo fisioterapêutico de mobilização precoce começou 12-18 horas após a ACTP. Um teste T não pareado bicaudal foi utilizado para as comparações, e p-valores $<0,05$ foram aceitos como significativos.

Resultados: Ao comparar os dois grupos, na alta, o GMP apresentou um aumento no SDNN $(23,55 \pm 12,05$ a 37,29 \pm $16,25 ; p=0,042)$, índice triangular $(8,99 \pm 3,03$ a $9,66 \pm 3,07 ; p=0,014)$ e VLF $(694,20 \pm 468,20$ a 848,37 $\pm 526,51$;

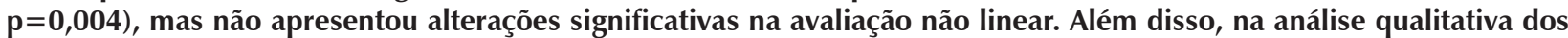
GR, observou-se um padrão mais difuso e menos geométrico no GMP, indicando maior variabilidade, enquanto no GC, notou-se um padrão geométrico mais alterado.

Conclusão: $O$ protocolo de mobilização precoce promove uma melhoria no comportamento autonômico, conforme avaliado por VFC e GR, e pode ser considerado um procedimento útil para a melhor recuperação de pacientes submetidos a ACTP.

Palavras-chave: Doenças Cardiovasculares/mortalidade; Infarto do Miocárdio; Deambulação Precoce/métodos; Exercícios; Sistema Nervoso Autônomo; Angioplastia.

\begin{abstract}
Background: Recurrence Plots (RP) enable a nonlinear analysis of Heart Rate Variability (HRV) and provide information on the Autonomic Nervous System (ANS).

Objectives: To evaluate whether early ambulation in patients undergoing Percutaneous Transluminal Coronary Angioplasty (PTCA) influences the quantitative and qualitative components of RP.

Methods: A total of 32 participants who underwent PTCA were divided into a Control Group (CG - no physical exercises) and an Early Ambulation Group (EAG - with physical exercises). Beat-to-beat heart rate was recorded using a heart rate monitor in both groups upon admission and discharge. The linear indices in the time and frequency domains were analyzed, and nonlinear indices were obtained through RP. The Early Ambulation Physical Therapy Protocol began 12-18 hours after PTCA. A two-tailed unpaired t-test was used for comparisons, and p-values $<0.05$ were accepted as significant.
\end{abstract}

Correspondência: Marilita Falangola Accioly •

Universidade Federal do Triangulo Mineiro - Departamento de Fisioterapia Aplicada - Av. Frei Paulino, 30. CEP 38025-180, Uberaba, MG - Brasil E-mail: marilitafisio@gmail.com

Artigo recebido em 08/04/2020, revisado em 06/12/2020, aceito em 27/01/2021

DOI: https://doi.org/10.36660/abc.20200296 
Results: When comparing both groups, upon discharge, EAG showed an increase in SDNN (23.55 \pm 12.05 to $37.29 \pm 16.25 ; p=0.042)$, Triangular Index (8.99 \pm 3.03 to $9.66 \pm 3.07 ; p=0.014)$, and VLF (694.20 \pm 468.20 to $848.37 \pm 526.51 ; p=0.004)$, but without significant changes in the nonlinear evaluation. In addition, in the qualitative analysis of RP, a more diffuse and less geometric pattern was observed in EAG, indicating greater variability, while in CG, an altered and more geometric pattern was noted.

Conclusion: The Early Ambulation Protocol promotes an improvement in autonomic behavior as evaluated by HRV and by RP, which can thus be considered a useful procedure for better recovery of patients undergoing PTCA.

Keywords: Cardiovascular Diseases/mortality; Myocardial Infarction; Early Ambulation/methods; Exercise; Autonomic Nervous System; Angioplasty.

Full texts in English - http://www.arquivosonline.com.br

\section{Introdução}

As doenças cardiovasculares são responsáveis pelo número mais alto de mortes em todo o mundo. ${ }^{1}$ Entre elas, o infarto do miocárdio agudo (IMA) é a principal causa de mortalidade no Brasil. ${ }^{2}$ Entretanto, a sobrevida desses pacientes aumentou devido a avanços tecnológicos, tais como a angioplastia coronária transluminal percutânea (ACTP). ${ }^{3,4}$

Em associação com a ACTP, a interação multidisciplinar desempenha um papel importante na redução da mortalidade, ${ }^{5}$ uma vez que a mobilização precoce evita o confinamento ao leito e seus vários efeitos deletérios, tais como o declínio funcional e a redução da qualidade de vida após a alta. ${ }^{6}$ Entretanto, ainda é muito comum que pacientes permaneçam confinados ao leito por receio de instabilidade hemodinâmica. ${ }^{7}$

Em contraste, a análise da modulação autonômica cardíaca pela variabilidade de frequência cardíaca (VFC) foi utilizada como preditor de riscos cardiovasculares em várias condições. ${ }^{8,9}$ Entretanto, a maioria dos estudos utilizou a análise linear da VFC. ${ }^{10-12}$

O corpo humano é uma boa ilustração de um "sistema complexo" caracterizado pela interação contínua de seus vários órgãos para manter a vida. Sua complexidade resulta em um modo de comportamento que é tipicamente não linear em situações normais. ${ }^{13}$ Assim, os métodos relacionados à dinâmica não linear são geralmente mais relevantes clinicamente para uma melhor interpretação do comportamento fisiopatológico da VFC sob várias condições, e, consequentemente, seu valor prognóstico, complementando as informações obtidas com avaliações tradicionais. ${ }^{14}$

Gráficos de recorrência (GR) são um método de análise não linear idealizado por Eckmann et al., ${ }^{15}$ que propõe a análise do comportamento de um sistema, representado por uma série temporal, em um espaço abstrato chamado espaço fásico, permitindo a quantificação e a qualificação da VFC. ${ }^{15,16}$

Além disso, pouco se sabe sobre respostas agudas ao exercício precoce na modulação autonômica e função cardiovascular no período pós-operatório imediato de pacientes submetidos a revascularização do miocárdio e ACTP. $^{17}$

Portanto, o presente estudo pretendeu avaliar se a mobilização precoce em pacientes submetidos a ACTP influencia os componentes quantitativos e qualitativos dos GR.

\section{Métodos}

\section{Amostra}

Esse é um ensaio clínico prospectivo, controlado e quase-experimental. A amostra incluiu indivíduos que foram submetidos a ACTP no Hospital de Clínicas da Universidade Federal do Triângulo Mineiro.

O tamanho da amostra foi calculado considerando-se a prevalência de indivíduos que precisam de angioplastia e estão hospitalizados em uma unidade de cuidado coronário. Para o cálculo, foi utilizada a seguinte fórmula: $n=Z^{2} \times p(p-100)$ / e ${ }^{2}$, em que "Z" é o valor crítico constante que corresponde ao intervalo de confiança de 95\% (IC95\%); "p" é a prevalência da doença/principal variável; e " $\mathrm{e}$ " é o erro de amostragem, que pode variar em até $10 \%$ do valor real da população selecionada para a amostra, sugerindo uma amostra de 15 indivíduos para cada grupo.

O fluxograma para recrutamento e seleção de amostra é apresentado na Figura 1.

A amostra foi composta de 32 participantes que atenderam aos seguintes critérios de inclusão: ter pelo menos 18 anos de idade; apresentar um diagnóstico médico de um IMA sem complicações (Killip I ou II), com ou sem supradesnivelamento do segmento ST e/ou indicação de ACTP eletiva (ACTP bemsucedida com estenose residual de menos de 50\%); ser capaz de entender as instruções para realizar exercícios físicos. Em seguida, os participantes foram divididos em dois grupos: Grupo de mobilização precoce (GMP) com 16 participantes submetidos ao protocolo de mobilização precoce; e grupo de controle (GC) com 16 participantes não submetidos ao protocolo de mobilização precoce. A amostra foi ajustada para idade, sexo e diagnóstico médico.

Indivíduos com pelo menos uma das características abaixo foram excluídos do estudo: histórico prévio de IMA, IMA com complicações (Killip III e IV), implante de marcapasso, bloqueio atrioventricular de $2^{\circ}$ ou 3 o grau, sequela de acidente vascular cerebral, amputação de membro inferior, estenose aórtica grave, cirurgia de revascularização do miocárdio prévio, insuficiência cardíaca, instabilidade hemodinâmica em repouso, piora da condição clínica geral, condição febril, insuficiência respiratória (necessidade de ventilação mecânica).

Os procedimentos do estudo seguiram todas as normas da Resolução no 466 do CNS e foi aprovado pelo Comitê de Ética e Pesquisa da Universidade Federal do Triângulo Mineiro sob o número de resolução 2.319.890 e pelo Registro Brasileiro de Ensaios Clínicos sob o número RBR-9w378x. 


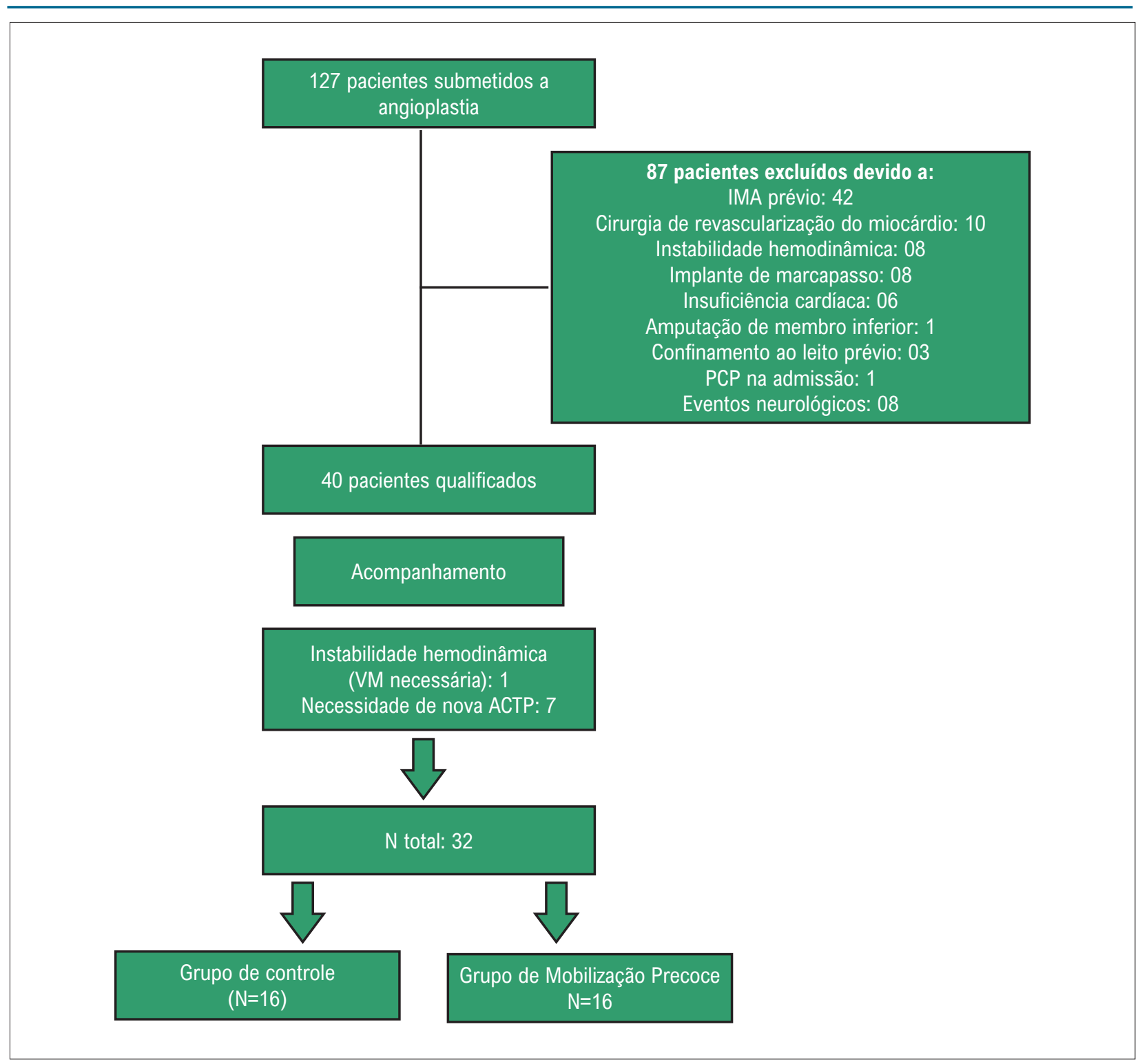

Figura 1 - Fluxograma mostrando o recrutamento e a seleção dos participantes do estudo. IMA: Infarto do miocárdio agudo. PCP: Parada cardiopulmonar ACTP: Angioplastia coronária transluminal percutânea. VM: Ventilação mecânica.

\section{Protocolo experimental}

O protocolo experimental consistiu em quatro fases. A fase I foi conduzida com entrevistas e avaliação de registros médicos. A fase II foi realizada de 12 a 18 horas após a ACTP e consistiu em registrar a frequência cardíaca batimento a batimento, utilizando um cardiofrequencímetro Polar ${ }^{\circledR}$ modelo RS800CX (Polar Kempele, Finlândia), e coletando o tacograma continuamente por 20 minutos. Durante todo o processo de coleta, o participante permaneceu em repouso, em posição supina, em silêncio e acordado. A fase III foi caracterizada pela implementação de um protocolo de mobilização precoce, realizado apenas pelo GMP.

$\mathrm{Na}$ fase IV, foi realizado um novo eletrocardiograma para a analisar a VFC, seguindo o mesmo procedimento realizado na fase II.

\section{Protocolo de Mobilização Precoce}

O Protocolo de Mobilização Precoce foi adaptado do protocolo utilizado no Grady Memorial Hospital e na Escola de Medicina da Emory University ${ }^{18}$ composto de passos progressivos em posições diferentes, conforme descrito na Tabela 1. O protocolo foi iniciado e aplicado nas posições de acordo com o status funcional do participante, que foi verificado por avaliações e comunicação com a equipe multidisciplinar. O GMP realizou o protocolo durante o período de hospitalização, que consistiu em duas sessões por dia (4 intervenções durante a permanência da UTI).

Os seguintes critérios foram utilizados para interromper o Protocolo de Mobilização Precoce: sinais e/ou sintomas de fadiga, dor no peito, dispneia, cianose, palidez, taquicardia (>120 batimentos por minuto), bradicardia, 


\begin{tabular}{|c|c|}
\hline Posição & Exercícios \\
\hline \multirow{6}{*}{ Supina } & A) Um set de exercícios de diafragma com movimento diagonal ativo-assistido ou ativo-livre do membro superior (10 vezes para cada membro); \\
\hline & B) Repetir o exercício por um set com ambos os membros simultaneamente (10 vezes); \\
\hline & C) Repetir o exercício por um set com ambos os membros simultaneamente (10 vezes); \\
\hline & D) Flexão tripla (10 vezes) + abdução/adução do quadril ativas-assistidas ou ativas-livres (10 vezes para cada membro); \\
\hline & E) Flexão-extensão de tornozelo (10 vezes) para cada pé; \\
\hline & E) Circundução de tornozelo (10 vezes) para cada pé; \\
\hline Sentado & Exercícios ativos de $\mathrm{A}$ a $\mathrm{F}$ \\
\hline \multirow{5}{*}{ Ortostatismo } & Exercícios ativos de A a F e ativos para membros inferiores (suportado, se necessário) \\
\hline & G) Ficar nas pontas dos pés (10 vezes) com ambos os membros simultaneamente; \\
\hline & H) Meio agachamento (10 vezes); \\
\hline & I) Marcha no lugar (por 30 segundos); \\
\hline & J) Deambulação no quarto (1 volta). \\
\hline
\end{tabular}

arritmias complexas e hipotensão (Pressão arterial média $<65 \mathrm{mmHg}$ ).

\section{Avaliação de modulação autonômica}

Para a análise de índices de VFC, registros de intervalo de RR foram transmitidos para um computador utilizando o software Polar Precision Performance (versão 4.01.029) ${ }^{19}$ e convertidos em arquivo de texto. Apenas as séries com mais de 95\% de batimentos sinusais foram analisadas, após a seleção dos 1000 pontos mais estáveis (Kubios HRV Software, versão 2.0, Universidade de Kuopio, Finlândia). Os dados foram filtrados usando o filtro padrão do software Polar Precision Performance (versão 4.01.029), com filtro moderado. Em seguida, uma ferramenta de filtragem computacional chamada T-RR Filter ${ }^{20}$ foi utilizada.

Entre os métodos lineares, o domínio tempo mede os intervalos RR normais (iR-R) e as várias medições são calculadas a partir desses intervalos, incluindo: desvio padrão do iR-R normal médio (SDNN), que corresponde a efeitos simpáticos e parassimpáticos, e representam variabilidade global; a porcentagem do iR-R adjacentes com diferença de duração acima de 50 milissegundos ( $\mathrm{NNN50);} \mathrm{e} \mathrm{a} \mathrm{raiz} \mathrm{quadrada} \mathrm{média}$ das diferenças sucessivas entre os $\mathrm{iR}-\mathrm{R}$ adjacentes usuais (RMSSD). As variáveis RMSSD e pNN50 estão relacionadas apenas ao comportamento parassimpático, enquanto o SDNN reflete todos os componentes responsáveis pela variabilidade. $\mathrm{O}$ índice triangular refere-se ao número de todos os iR-R dividido pela frequência desses $\mathrm{iR}-\mathrm{R}$ no compartimento modal do histograma, refletindo, dessa forma, a variabilidade global. ${ }^{21}$

Para a análise do domínio frequência, o método de interpolação por spline cúbica em $4 \mathrm{~Hz}$ foi aplicado, e a densidade espectral de potência do segmento mais estável foi calculada utilizando-se a Transformação rápida de Fourier (FFT), calculando, em milissegundos ao quadrado $\left(\mathrm{ms}^{2}\right)$, os componentes espectrais para banda de frequência muito baixa (VLF) $(<0,04 \mathrm{~Hz})$, frequência baixa (LF) $(0,04-0,15 \mathrm{~Hz})$, e frequência alta $(\mathrm{HF})(0,15$ a 0,40 Hz), além da relação entre esses componentes (LF/HF).
LF representa a modulação parassimpática; HF representa a atividade de modulação vagal. ${ }^{22}$ VLF reflete regulações humorais, vasculares, térmicas, bem como a atividade do sistema renina-angiotensina-aldosterona. ${ }^{22}$ Além disso, a relação entre LF e HF pode ser uma medida de balanço simpatovagal. ${ }^{22,23}$

Os GR podem ser usados para análise não linear de VFC, que foi realizada de maneira qualitativa e quantitativa. A análise qualitativa foi realizada pela visualização do padrão gráfico e a análise quantitativa utilizou os seguintes índices: Recorrência (REC), Determinismo (DET), Entropia de Shannon (ES), Laminaridade (LAM), Tempo de permanência (TT) e Comprimento máximo de linha (MaxLine). ${ }^{15}$ Os seguintes parâmetros foram usados nos GR: dimensão de incorporação $=10$; atraso $=1$, raio $=70$, linha $=2^{24}$ e esquema de cores $=$ Volcano.

É utilizada uma série temporal para a construção do GR. De acordo com faixas de valores entre medidas (dimensões) e distâncias ou intervalos de tempo (raio), pode-se verificar se existem valores de recorrência ou não. O uso de cores diferentes representa vários raios, complementando a aparência visual típica dos GR. ${ }^{24}$

Os GR podem ser analisados por padrões de pequena ou grande escala. Os padrões de pequena escala se encaixam em pontos, e linhas diagonais e verticais, o que permite uma análise qualitativa. Por exemplo, em indivíduos saudáveis, o GR mostra uma linha diagonal e menos quadrados aparentes, indicando uma VFC mais alta. Em indivíduos com alguma deficiência na modulação autonômica, o GR mostra quadrados definidos no gráfico, mais formas geométricas, indicando a periodicidade inerente, comportamento linear e VFC baixa. ${ }^{24,25}$

$\mathrm{Na}$ análise quantitativa, alguns índices são gerados: índice de recorrência (REC\%), que quantifica a porcentagem de pontos recorrentes com um raio específico; porcentagem de determinismo (DET\%), representando as linhas diagonais formadas pelos pontos de recorrência; comprimento médio das linhas diagonais (Lmean) e comprimento máximo das 
linhas diagonais (Lmax), representando a maior diagonal exceto a principal; laminaridade (LAM), que são os pontos de recorrência que formam linhas verticais; tempo de permanência (TT), que é o comprimento médio das linhas verticais; entropia, representando a entropia de Shannon, que mede a complexidade de distribuição de linhas diagonais. Nesse caso, diferentemente de outras interpretações, entende-se que quanto mais alta a entropia de Shannon, mais linear será a série temporal. ${ }^{25}$

Para a análise qualitativa, sistemas caóticos, aleatórios, periódicos e lineares construídos por meio da fórmula matemática relatada por Takakura et. al. ${ }^{24}$ foram usados como modelos de GR, conforme mostrado na Figura 2.

\section{Análise estatística}

Foi realizada a análise descritiva de variáveis categóricas e contínuas. A presença ou ausência de distribuição normal de variáveis foi avaliada por testes de Shapiro-Wilk. Dados contínuos com distribuição gaussiana foram expressos como média \pm desvio padrão. Variáveis categóricas foram expressas como valores absolutos e porcentagens.

Um teste T não pareado bicaudal foi definido para análises intergrupos, admitindo-se um erro alfa de 5\%. Todas as análises foram realizadas com o software Stats Direct Statistical versão 3.3.3.

\section{Resultados}

A amostra analisada foi homogênea em relação a diagnóstico clínico, pressão arterial sistêmica, frequência cardíaca em repouso, duração da internação, número de stents implantados, e índices de VFC. A tabela 2 descreve a caraterização das amostras de ambos os grupos.

Os medicamentos usados pelo GC foram betabloqueadores (81,3\%), hipolipemiantes (62,5\%), IECA (50\%), Aspirina (62,5\%) e Ticlopidina/clopidogrel $(18,8)$; enquanto o GMP utilizou $(87,5 \%)$, hipolipemiantes (53,6\%), IECA (50\%), Aspirina (62,5\%) e Ticlopidina/clopidogrel (37,5\%).

A tabela 3 resume a análise de índices lineares pelos domínios de tempo e frequência, em que se pode observar uma diferença estatisticamente significativa no SDNN, índice triangular e VLF, ao comparar GC e GMP. A Tabela 4 mostra a análise quantitativa de índices não lineares pelo software Visual Recurrence Analysis.
Não há diferenças estatísticas entre ambos os momentos, ao comparar GC e GMP.

Entretanto, uma piora geral do padrão do Grupo de controle pode ser observada na Figura 3, que mostra a análise qualitativa do GR. Na alta, há um número maior de quadrados escuros e mais formas geométricas, indicando linearidade mais alta, e, portanto, menos homeostase do sistema, quando comparado com a admissão. Além disso, ao comparar GMP e GC, observase um padrão caótico, com menos quadrados aparentes e mais homogêneos no GMP, indicando maior complexidade e melhoria da homeostase.

\section{Discussão}

Os principais achados da análise intragrupo do GMP foram o aumento significativo dos índices lineares (SDNN e pNN50) na alta, quando comparados aos da admissão. Na análise intergrupo do GMP, observa-se um padrão mais complexo e caótico.

O aumento de índices lineares é indicativo de VFC alta, representada pelo aumento da atividade parassimpática em pNN50, e atividade autonômica global em SDNN, após a aplicação do Protocolo de Mobilização Precoce. Esse resultado implica a melhoria da saúde global do sistema cardiovascular, considerando que o aumento da atividade parassimpática está relacionado à VFC mais alta, e à mortalidade cardiovascular mais baixa, ${ }^{26,27}$ demonstrando a influência benéfica dos exercícios realizados na UTI.

Na análise intergrupo, os índices de SDNN, Índice triangular, e VLF demonstram uma diferença estatisticamente significativa na alta. Os índices de SDNN implicam as informações globais na modulação simpática e parassimpática; o Índice triangular expressa variabilidade no intervalo de RR, intimamente relacionada a SDNN; e a VLF está relacionada ao sistema angiotensina-aldosterona renina, termorregulação, e tônus vasomotor periférico. ${ }^{25}$

Alguns estudos ${ }^{27,28}$ demonstraram evidência de que a mobilização precoce aumenta a modulação autonômica em indivíduos após o IMA. Entretanto, esses estudos utilizaram métodos lineares em suas análises, e estudos recentes indicam que o corpo humano tem um comportamento não linear. Portanto, a análise da modulação autonômica por métodos não lineares é crucial. ${ }^{14}$

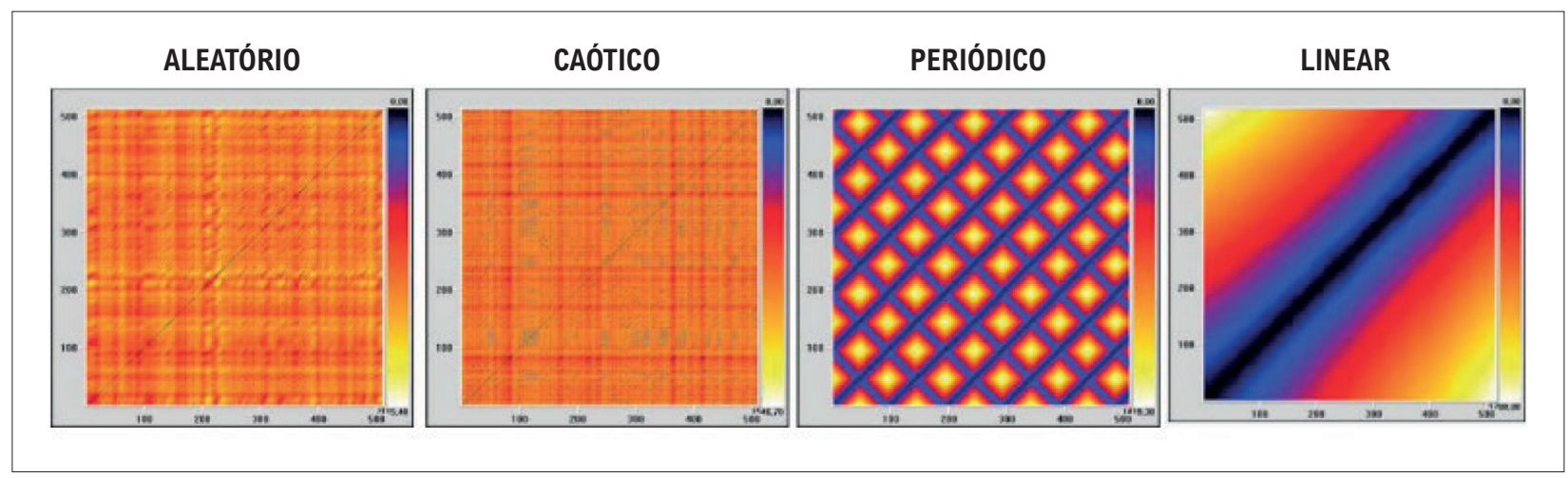

Figura 2 - GR de sistemas aleatórios, caóticos, periódicos e lineares obtidos com fórmulas matemáticas. Fonte: Takakura et al., ${ }^{21}$ 


\begin{tabular}{|c|c|c|c|}
\hline & Grupo de controle $(N=16)$ & $\begin{array}{l}\text { Grupo de Mobilização Precoce } \\
\qquad(\mathrm{N}=16)\end{array}$ & p-valor* \\
\hline \multicolumn{4}{|l|}{ Perfil sociodemográfico } \\
\hline Idade: anos & $61,00 \pm 8,34$ & $63,50 \pm 9,64$ & 0,439 \\
\hline Sexo: masculino (n) & $08(50 \%)$ & $08(50 \%)$ & \\
\hline IMC $\left(\mathrm{kg} / \mathrm{m}^{2}\right)$ & $27 \pm 3$ & $29 \pm 3$ & 0,467 \\
\hline \multicolumn{4}{|l|}{ Diagnóstico } \\
\hline Doença Arterial Coronariana (n) & $08(50 \%)$ & $08(50 \%)$ & \\
\hline Infarto do Miocárdio Agudo (n) & $08(50 \%)$ & $08(50 \%)$ & \\
\hline \multicolumn{4}{|l|}{ PA/FC (em repouso) } \\
\hline Frequência Cardíaca (bpm) & $71,38 \pm 11,09$ & $71,19 \pm 10,08$ & 0,960 \\
\hline Pressão arterial sistólica (mmHg) & $130,25 \pm 22,62$ & $134,38 \pm 19,26$ & 0,583 \\
\hline Pressão arterial diastólica $(\mathrm{mmHg})$ & $76,50 \pm 17,29$ & $72,06 \pm 9,95$ & 0,383 \\
\hline Pressão arterial média (mmHg) & $94,06 \pm 21,22$ & $92,69 \pm 13,05$ & 0,827 \\
\hline \multicolumn{4}{|l|}{ Obstrução coronária } \\
\hline Artéria coronária direita (n) & $12(75 \%)$ & $10(62,5 \%)$ & \\
\hline Anterior descendente $(n)$ & $8(50 \%)$ & $8(50 \%)$ & \\
\hline Circunflexa (n) & $2(12,5 \%)$ & $3(18,8 \%)$ & \\
\hline Duração da internação (dias) & $3,94 \pm 1,48$ & $3,75 \pm 1,18$ & 0,113 \\
\hline Número de stents & $1,63 \pm 0,71$ & $1,94 \pm 0,85$ & 0,215 \\
\hline
\end{tabular}

N: número de participantes; média \pm desvio padrão; IMC: indice de massa corporal; kg/m2; quilograma por metro quadrado; PA: pressão arterial; FC: frequência cardiaca; bpm: batimentos por minuto; $\mathrm{mmHg}$ : milimetros de mercúrio. * teste $t$ de Student não pareado.

Tabela 3 - Variabilidade de Frequência Cardíaca analisada por Métodos Lineares: Controle vs. Mobilização Precoce

\begin{tabular}{|c|c|c|c|c|c|c|}
\hline \multirow{2}{*}{ Índices de VFC } & \multicolumn{2}{|c|}{ Controle } & \multicolumn{2}{|c|}{ Mobilização Precoce } & \multicolumn{2}{|c|}{$\begin{array}{c}\text { Controle x Mobilização } \\
\text { Precoce }\end{array}$} \\
\hline & Admissão & Alta & Admissão & Alta & Admissão & Alta \\
\hline Domínio tempo & Média (DP) & Média (DP) & Média (DP) & Média (DP) & p-valor & p-valor \\
\hline RR média & $894,58 \pm 112,65$ & $825,29 \pm 85,04$ & $890,60 \pm 147,14$ & $908,12 \pm 140,25$ & 0,9321 & 0,0544 \\
\hline SDNN & $27,73 \pm 18,91$ & $27,4 \pm 8,71$ & $23,55 \pm 12,05$ & $37,29 \pm 16,25$ & 0,4610 & $0,0428^{*}$ \\
\hline RMSSD & $21,98 \pm 11,10$ & $15,69 \pm 9,09$ & $19,68 \pm 11,03$ & $20,48 \pm 13,47$ & 0,5601 & 0,2482 \\
\hline PNN50 & $4,19 \pm 7,65$ & $3,83 \pm 6,85$ & $2,30 \pm 5,26$ & $5,12 \pm 6,53$ & 0,7046 & 0,4864 \\
\hline Índice triangular & $9,34 \pm 4,99$ & $7,23 \pm 2,09$ & $8,99 \pm 3,03$ & $9,66 \pm 3,07$ & 0,8126 & $0.0148^{*}$ \\
\hline TINN & $142,81 \pm 54,25$ & $131,25 \pm 39,39$ & $120,62 \pm 63,03$ & $130,0 \pm 70,75$ & 0,2944 & 0,9513 \\
\hline \multicolumn{7}{|l|}{ Domínio Frequência } \\
\hline Potência de VLF $\left(\mathrm{ms}^{2}\right)$ & $927,23 \pm 1395,20$ & $407,56 \pm 237,61$ & $694,20 \pm 468,20$ & $848,37 \pm 526,51$ & 0,5308 & $0,0043^{*}$ \\
\hline Potência de LF $\left(\mathrm{ms}^{2}\right)$ & $300,06 \pm 367,49$ & $177,81 \pm 154,94$ & $233,93 \pm 165,23$ & $380,50 \pm 513,86$ & 0,5165 & 0,1414 \\
\hline Potência de HF $\left(\mathrm{ms}^{2}\right)$ & $148,43 \pm 174,09$ & $159,87 \pm 223,07$ & $94,12 \pm 117,58$ & $177,68 \pm 159,76$ & 0,4677 & 0,3282 \\
\hline Potência de LF/HF $\left(\mathrm{ms}^{2}\right)$ & $2,86 \pm 2,12$ & $3,39 \pm 2,49$ & $2,80 \pm 1,61$ & $3,94 \pm 3,65$ & 0,9295 & 0,6205 \\
\hline
\end{tabular}




\begin{tabular}{|c|c|c|c|c|c|c|}
\hline \multirow{3}{*}{ Índices de VFC } & \multicolumn{2}{|c|}{ Controle } & \multicolumn{2}{|c|}{ Mobilização Precoce } & \multicolumn{2}{|c|}{ Controle x Mobilização Precoce } \\
\hline & Admissão & Alta & Admissão & Alta & Admissão & Alta \\
\hline & Média (DP) & Média (DP) & Média (DP) & Média (DP) & p-valor & p-valor \\
\hline \multicolumn{7}{|l|}{ Visual Recurrence Analysis (VRA) } \\
\hline Média & $890,64 \pm 147,15$ & $908,22 \pm 140,36$ & $893,71 \pm 101,13$ & $843,18 \pm 90,72$ & 0,9457 & 0,1318 \\
\hline Porcentagem de recorrência & $40,71 \pm 7,56$ & $40,18 \pm 2,41$ & $38,54 \pm 3,15$ & $43,60 \pm 13,66$ & 0,3021 & 0,3389 \\
\hline Porcentagem de determinismo & $86,35 \pm 10,99$ & $86,65 \pm 11,37$ & $83,71 \pm 13,78$ & $86,63 \pm 11,39$ & 0,553 & 0,8099 \\
\hline Porcentagem de laminaridade & $92,38 \pm 6,29$ & $91,05 \pm 9,61$ & $89,62 \pm 9,66$ & $91,51 \pm 9,77$ & 0,3477 & 0,8941 \\
\hline Tempo de permanência & $11,65 \pm 12,15$ & $8,73 \pm 3,11$ & $7,68 \pm 3,67$ & $7,82 \pm 2,40$ & 0,2271 & 0,3668 \\
\hline Relação & $2,14 \pm 0,23$ & $2,12 \pm 0,22$ & $2,16 \pm 0,25$ & $2,14 \pm 0,28$ & 0,8472 & 0,8257 \\
\hline Entropia & $3,79 \pm 0,75$ & $3,83 \pm 0,52$ & $3,66 \pm 0,60$ & $3,71 \pm 0,48$ & 0,5886 & 0,5117 \\
\hline MaxLine & $219,93 \pm 191,23$ & $241,68 \pm 223,29$ & $238,12 \pm 166,34$ & $171,12 \pm 95,56$ & 0,7761 & 0,2587 \\
\hline
\end{tabular}

Dados expressos como média (desvio padrão) *: diferença estatisticamente significativa $(p<0,05)$. Teste $T$ pareado (amostras paramétricas) e teste de Wilcoxon (amostra não paramétrica). teste T de Student não pareado (Controle x Mobilização Precoce).

A análise não linear, diferentemente da análise linear, mede qualidade, escala e correlação de propriedades de sinais, proporcionando, dessa forma, uma interpretação de imprevisibilidade, complexidade e fractalidade do sistema. ${ }^{14}$ Meyerfeldt et al., ${ }^{26}$ identificaram "sensibilidade" mais alta de análise não linear comparada à análise em comportamentos patológicos, tais como as taquiarritmias.

Ao analisar os resultados de índices para domínios de caos, em valores absolutos, não foram encontradas diferenças estatisticamente significativas. Entretanto, ao analisar o aspecto qualitativo do GR (Figura 3), é possível observar que o GR dos participantes tratados com mobilização precoce apresenta melhoria da VFC, já que apresentam cores mais heterogêneas e menos padrões geométricas. Comparados a modelos matemáticos (Figura 2), eles apresentam um comportamento que tendem ao caos, indicando maior variabilidade e, portanto, melhoria autonômica. O estudo realizado por Manata et al. ${ }^{30}$ corroboram os achados deste estudo, que utilizaram GR para comparar indivíduos saudáveis a indivíduos com doença pulmonar obstrutiva crônica (DPOC). Os autores concluíram que indivíduos portadores de DPOC têm VFC mais baixa, já que o GR apresentou quadrados mais visíveis e uma configuração mais homogênea. Também se observaram pontos de recorrência mais altos na comparação com indivíduos saudáveis, o que indica um sistema mais recorrente e menos dinâmico, e menos modulação autonômica complexa nessa população.

Godoy \& Gregório $^{31}$ ao analisar o GR de vários grupos, conseguiram verificar uma diferença na variabilidade desses indivíduos (recém-nascidos, adultos, portadores de doenças renais, e indivíduos com morte cerebral declarada), para as análises quantitativas e qualitativas de GR. A evidência parece corroborar nossos achados, já que foi observada a influência positiva da mobilização precoce na VFC na análise qualitativa de GR. Entretanto, não foram observadas diferenças estatísticas na análise quantitativa.
Takakura et al., ${ }^{21}$ analisaram GR em pacientes que foram submetidos a transplante cardíaco, e observaram sinais quantitativos e qualitativos de recuperação de VFC, demonstrando que a reinervação autonômica cardíaca iniciou gradualmente após o transplante.

Acredita-se que o pequeno número de intervenções do Protocolo de Mobilização Precoce (apenas 4 intervenções) pode ter contribuído para a ausência de uma diferença estatisticamente significativa na análise quantitativa. É possível que um número mais alto de intervenções promova a melhoria da modulação autonômica em outros índices de VFC. Isso pode ser considerado um fator limitador deste estudo.

Para esta pesquisa, somente foram utilizados estudos que analisaram o GR após procedimentos cirúrgicos e/ou doenças, e não foram identificados estudos que analisaram os efeitos agudos do exercício físico nos pacientes com IMA ou Doença arterial coronariana (DAC). Isso sugere que são necessárias pesquisa adicionais para analisar a dinâmica de VFC não linear em relação a vários protocolos de mobilização precoce.

\section{Conclusão}

O protocolo de mobilização precoce promove uma melhoria no comportamento autonômico, conforme avaliado por variabilidade de frequência cardíaca e por gráficos de recorrência, e pode ser considerado um procedimento útil para a melhor recuperação de pacientes submetidos a angioplastia coronária transluminal percutânea.

\section{Contribuição dos autores}

Concepção e desenho da pesquisa: Neri GPO, Gregório ML, Godoy MF, Accioly MF; Obtenção de dados: Silveira BO, Melo JL, Neri GPO; Análise e interpretação dos dados: Silveira BO, Melo JL, Gregório ML, Godoy MF, Accioly MF; Análise estatística: Gregório ML, Godoy MF, Accioly MF; Redação do manuscrito: Silveira BO, Melo JL, Accioly MF; 

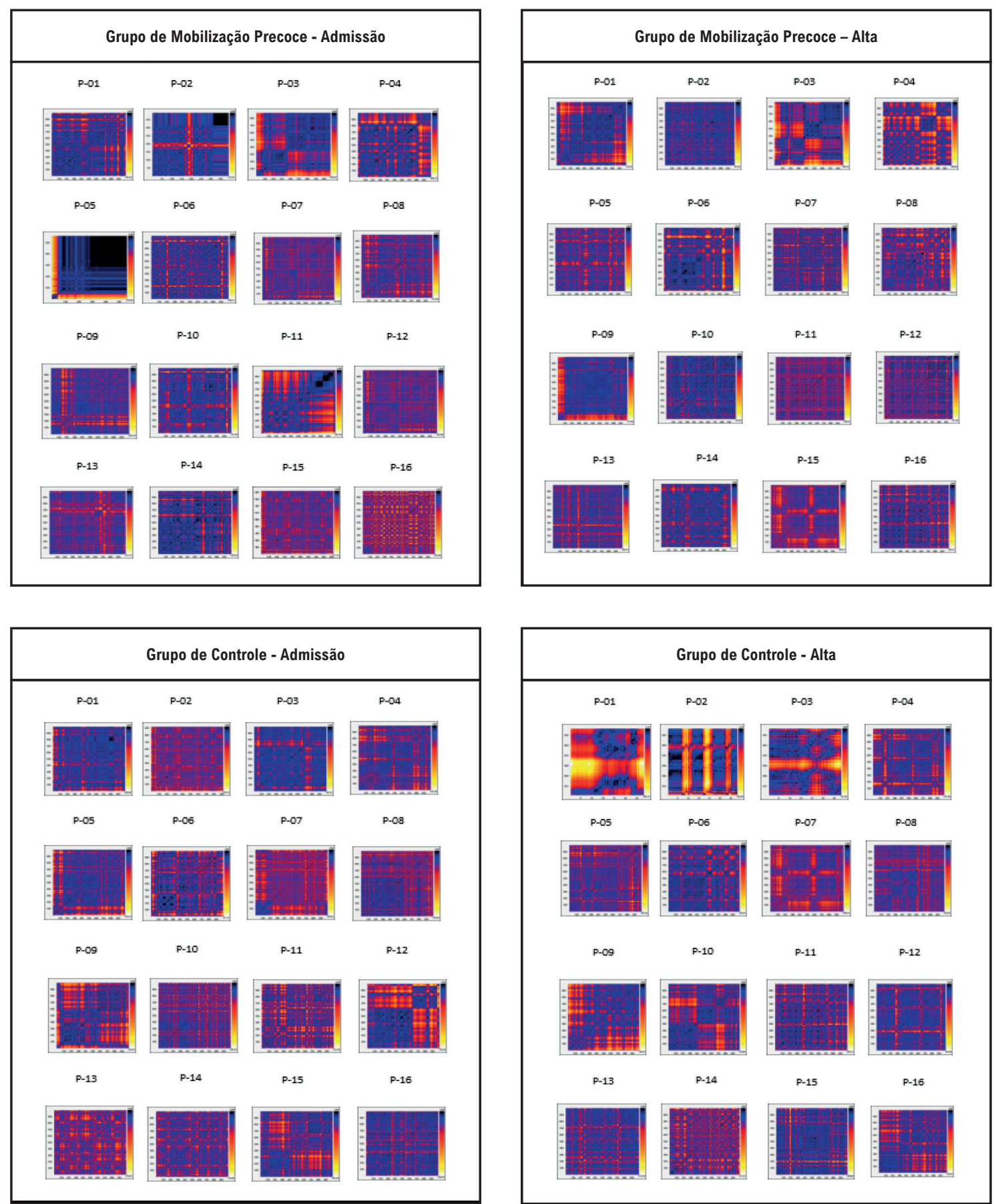

Figura 3 - Gráficos de recorrência de participantes identificado por P_00 admissão e alta. Fonte: Pesquisa.

Revisão crítica do manuscrito quanto ao conteúdo intelectual importante: Silveira BO, Melo JL, Gregório ML, Godoy MF, Accioly MF.

\section{Potencial conflito de interesse}

Não há conflito com o presente artigo

\section{Fontes de financiamento}

O presente estudo não teve fontes de financiamento externas.

\section{Vinculação acadêmica}

Este artigo é parte de dissertação de mestrado de Gaziella P. O Neri pelo Universidade Federal do Triângulo Mineiro. 


\section{Referências}

1. Feres F, Costa RA, Siqueira D, Costa Jr JR, Chamié D, Staico R, et al. Diretriz da Sociedade Brasileira de Cardiologia e da Sociedade Brasileira de Cardiologia Intervencionista sobre Intervenção Coronária Percutânea. Arq Bras Cardiol,2017; v.109(1Supl.1):1-81.

2: França EET, Ferrari F, Fernandes P, Cavalcanti R, Duarte A, Martinez BP et al . Fisioterapia em pacientes críticos adultos: recomendações do Departamento de Fisioterapia da Associação de Medicina Intensiva Brasileira. Rev Bras Ter Intensiva. 2012 Mar; 24( 1 ): 6-22. https://doi. org/10.1590/S0103-507X2012000100003.

3. Herdy AH, López-Jiménez F, Terzic CP, Milani M, Stein R, Carvalho T, et al. Diretriz Sul-Americana de Prevenção e Reabilitação Cardiovascular. Arq Bras Cardiol.2014;103(2 Supl 1):1-31.

4. Umeda IIK. Manual de Fisioterapia na Reabilitação Cardiovascular. São Paulo: Manole. p.46-68. ISBN: 9788520424872.

5. Bauernschmitt R, Malberg H, Wessel N, Kopp B, Schirmbeck EU, Lange R. Impairment of cardiovascular autonomic control in patients early after cardiac surgery. Eur J Cardiothorac Surg. 2004 Mar;25(3):320-6. https:// doi.org/10.1016/j.ejcts.2003.12.019

6. Kunz VC, Souza RB, Takahashi ACM, Catai AM, Silva E. The relationship between cardiac autonomic function and clinical and angiographic characteristics in patients with coronary artery disease. Braz J Phys Ther. 2011; 15(6):503-10. https://doi.org/10.1590/S141335552011005000020 .

7. Wolf MM, Varigos GA, Hunt D, Sloman JG. Sinus arrhythmia in acute myocardial infarction. Med J Austr. 1978;2(2):52-3. https://doi. org/10.5694/j.1326-5377.1925.tb11693.x

8. Kleiger RE, Miller JP, Bigger JT Jr, Moss AJ. Decreased heart rate variability and its association with increased mortality after acute myocardial infarction. Am J Cardiol. 1987;59(4):256-62. https://doi. org/10.1016/0002-9149(87)90795-8.

9. Erdogan A, Coch M, Bilgin M, Parahuleva M, Tillmanns H, Waldecker B, Soydan N. Prognostic value of heart rate variability after acute myocardial infarction in the era of immediate reperfusion. Herzschrittmachertherapie \& Elektrophysiologie. 2008;19:161-8. https://doi.org/10.1007/s00399008-0024-3

10. Beckers F, Verheyden B, Aubert AE. Aging and nonlinear heart rate control in a healthy population. Am J Physiol Heart Circ Physiol. 2006;290(6):H2560-70. https://doi.org/10.1152/ajpheart.00903.2005.

11. Godoy MF. Nonlinear Analysis of Heart Rate Variability: A Comprehensive Review. J Cardiol Ther. 2016;3(3):528-33. https://doi.org/10.17554/j. issn.2309-6861.2016.03.101-4

12. Eckmann, JP, Kamphorst, SO, Ruelle, D. "Recurrence Plots of Dynamical Systems", Europhys Lett. 187;4:973-7. https://doi.org/10.12691/bse-21-3.

13. Francesco B, Maria Grazia B, Emanuele G, Valentina F, Sara C, Chiara F, et al. Linear and nonlinear heart rate variability indexes in clinical practice. Computational and mathematical methods in medicine. 2012; (issue special): 1-5. https://doi.org/10.1155/2012/219080.

14. Barbosa P, Santos FV, Neufeld PM, Bernardelli GF, Castro SS, Fonseca JHP, et al.Efeitos da mobilização precoce na resposta cardiovascular e autonômica no pós-operatório de revascularização do miocárdio. ConScientiae Saúde. 2010;9(1):111-8. https://doi.org/10.1590/S0103-507X2012000100011.

15. Shved M, Tsuglevych L, Kyrychok I, Levytska L, Boiko T, Kitsak, Y. Cardioreabilitation peculiarities and correction of violations of sistolic, diasolic function and heart rate variability in patients with acute coronary syndrome and coronary artery revascularization. Galician Med J. 2017;24(4):E201749 doi: 1.21802/gmy.2017.4.9.

16. Quintana DS, Heathers JA, Kemp AH. On the validity of using the Pola RS800 heart rate monitor for heart rate variability research. Eur J Appl Physiol. 2012;112(12):4179-80. https://doi.org/10.1007/s00421-0122453-2.

17. Santos L, Pillat V, de Godoy MF, Barbosa CL, Barroso J, Macau E. T-RR Filter: Ferramenta computacional gráfica no uso do filtro adaptativo para análise da variabilidade da frequência cardíaca. Proceeding Series of the Brazailian Society of Computacional and Applied Mathematics. 2016;4(1):1133-9. https://doi.org/10.5540/03.2016.004.01.0096

18. TASK FORCE. Heart rate variability: standards of measurement, physiological interpretation and clinical use. Task Force of the European Society of Cardiology and the North American Society of Pacing and Electrophysiology. Circulation. 1996; 93(5):1043-65. https://doi.org/10.1161/01. CIR.93.5.1043

19. Hernando D, Garatachea D, Almeida R, Casajus J, Bailon R. Validation of heart rate monitor polar RS800 for heart rate variability analysis during exercise.J Strength Conditioning Res 2018;32(3):716-25. https://doi. org/10.1519/JSC.0000000000001662

20. Munk, PS, Butt, N, Larsen, AI. High-intensity interval exercise training improves heart rate variability in patients following percutaneous coronary intervention for angina pectoris. International J Int Cardiol.2010. 145(2):312-4. https://doi.org/31214.10.1016/j.ijcard.2009.11.015

21. Takakura IT, Hoshi RA, Santos MA, Pivatelli FC, Nóbrega JH, Guedes DL et al . Recurrence Plots: a New Tool for Quantification of Cardiac Autonomic Nervous System Recovery after Transplant. Braz. J. Cardiovasc Surg. 2017 ;32(4):245-52. https://doi.org/10.21470/1678-9741-20160035 .

22. Ferreira LL, de Souza NM, Bernardo AFB, Vitor ALR, Valenti VE, Vanderle LCM. Heart rate variability as a resource in physical. Fisioter Mov. 2013;26(1):25-36. https://doi.org/10.1590/S0103-51502013000100003.

23. Vanderlei LC, Pastre CM, Hoshi RA, Carvalho TD, Godoy MF. Basic notion of heart zate variability and its clinical applicability. Rev Bras Cir Cardiovasc. 2009;24(2):205-17. https://doi.org/10.1590/s0102-76382009000200018.

24. Hiss MDBS, Neves, VR, Hiss FC, Silva E, Silva AB, Catai AM. Segurança da intervenção fisioterápica precoce após o infarto agudo do miocárdio. Fisioter. Mov. 2012;25(1):153-63. https://doi.org/10.1590/S010351502012000100015.

25. Selig, FA, Tonolli ER, Silva EVCM, Godoy MF. Variabilidade da frequência cardíaca em neonatos prematuros e de termo. Arq. Bras. Cardiol. 2011 June; 96 (6): 443-449. https://doi.org/10.1590/S0066782X2011005000059.

26. Meyerfeldt U, Wessel N, Schutt H, Selbig D, Schumann A, Voss A, Kurths J, Ziehmann C, Dietz R, Schirdewan A. Heart rate variability before the onset of ventricular tachycardia: differences between slow and fast arrhythmias. Int J Cardiol. 2002;8(2-3):141-51. https://doi.org/10.1016/s01675273(02)00139-0.

27. Vanzella L.Bernardo AFB, Carvalho TD, Vanderlei F, Silva AK, Vanderle LC. Complexidade do sistema nervoso autônomo em indivíduos com DPOC. J Bras Pneumol.2018;44(1):24-30. https://doi.org/10.1590/s18067562017000000086

28. Godoy MF, Gregório ML. Diagnostic Relevance of Recurrence Plots for the Characterization of Health, Disease, or Death in Humans. J Hum Growth Dev. 2019;29(1):39-44. https://doi.org/10.7322/jhgd.157746. 